\title{
MULTIFRACTIONAL BROWNIAN FIELDS INDEXED BY METRIC SPACES WITH DISTANCES OF NEGATIVE TYPE
}

\author{
JACQUES ISTAS ${ }^{1}$
}

\begin{abstract}
We define multifractional Brownian fields indexed by a metric space, such as a manifold with its geodesic distance, when the distance is of negative type. This construction applies when the Brownian field indexed by the metric space exists, in particular for spheres, hyperbolic spaces and real trees.
\end{abstract}

Mathematics Subject Classification. 60G18, 60G15, 60G52.

Received June 16, 2010. Revised July 19, 2011.

\section{INTRODUCTION}

The concept of self-similarity is often used to give a mathematical meaning to the heuristic concept of roughness. In this domain the fractional Brownian motion $[6,13]$ of index $H$ is certainly the most famous model. The roughness index $H$ is constant and this is too restrictive for some applications. The multifractional Brownian motions associated with a function $H().[2,3,14]$ have therefore been defined for a while. The same has been done in higher dimensions for the multifractional fields indexed by Euclidean spaces [7]. Some data (texture, natural scene, geostatistical data, cosmic microwave background, ...) are indexed not by an Euclidean space but by a manifold. [10,11] has therefore defined fractional Brownian fields indexed by a metric space, such as a manifold equipped with its geodesic distance. It is then natural to define multifractional Brownian fields indexed by a metric space and this is the purpose of this paper.

\section{Multifractional Brownian fields indexed By EuClidean spaces}

\subsection{Euclidean spaces $\mathbb{R}^{n}$}

Classically $[2,3,7,14]$ the multifractional Brownian field $B_{n}$, indexed by $\mathbb{R}^{n}$ and associated with a multifractional function $H: \mathbb{R}^{n} \mapsto(0,1)$ is defined, up to a constant, as the centered Gaussian field with covariance

$$
R_{n}\left(X, X^{\prime}\right)=\int_{\mathbb{R}^{n}} \frac{\left(1-\mathrm{e}^{\mathrm{i}\langle X, Y\rangle}\right)\left(1-\mathrm{e}^{-\mathrm{i}\left\langle X^{\prime}, Y\right\rangle}\right)}{\|Y\|^{n+H(X)+H\left(X^{\prime}\right)}} \mathrm{d} Y,
$$

where $\langle X, Y\rangle$ stands for the Euclidean scalar product on $\mathbb{R}^{n}$, and $\|X\|^{2}=\langle X, X\rangle$.

Keywords and phrases. Fractional Brownian motion, self-similarity, complex variations, $H$-sssi processes.

1 Laboratoire Jean Kuntzmann, Université de Grenoble et CNRS, 38041 Grenoble Cedex 9, France. Jacques.Istas@imag.fr 
Proposition 2.1. Function $R$ can be written as:

$$
\begin{aligned}
R_{n}\left(X, X^{\prime}\right)= & \frac{C_{n}\left(H(X)+H\left(X^{\prime}\right)\right)}{2}\left(\|X\|^{H(X)+H\left(X^{\prime}\right)}+\left\|X^{\prime}\right\|^{H(X)+H\left(X^{\prime}\right)}\right. \\
& \left.-\left\|X-X^{\prime}\right\|^{H(X)+H\left(X^{\prime}\right)}\right) \\
C_{n}(\alpha)= & \frac{2 \pi^{(n+1) / 2} \Gamma((\alpha+1) / 2)}{\alpha \sin (\alpha \pi / 2) \Gamma(\alpha) \Gamma((n+\alpha) / 2)},
\end{aligned}
$$

where $\Gamma$ is the Gamma function.

Proof. Let $\alpha \in(0,2)$. Set

$$
I_{n}(X)=\int_{\mathbb{R}^{n}} \frac{\left|1-\mathrm{e}^{\mathrm{i}\langle X, Y\rangle}\right|^{2}}{\|Y\|^{n+\alpha}} \mathrm{d} Y .
$$

The change of variable $Z=\|X\| Y$ leads to

$$
I_{n}(X)=\|X\|^{\alpha} \int_{\mathbb{R}^{n}} \frac{\left|1-\mathrm{e}^{\mathrm{i}\langle X /\|X\|, Z\rangle}\right|^{2}}{\|Z\|^{n+\alpha}} \mathrm{d} Z .
$$

Let $\rho$ be a rotation such that $X /\|X\|=\rho((1,0, \ldots, 0))$. The change of variable $Z=\rho(\tilde{Z})$ leads to

$$
I_{n}(X)=\|X\|^{\alpha} \int_{\mathbb{R}^{n}} \frac{\left|1-\mathrm{e}^{\mathrm{i}\langle(1,0, \ldots, 0), \tilde{Z}\rangle}\right|^{2}}{\|\tilde{Z}\|^{n+\alpha}} \mathrm{d} \tilde{Z} .
$$

The integral $C_{n}[\alpha)=\int_{\mathbb{R}^{n}} \frac{\left|1-\mathrm{e}^{\mathrm{i}\langle(1,0, \ldots, 0), \tilde{Z}\rangle}\right|^{2}}{\|\tilde{Z}\|^{n+\alpha}} \mathrm{d} \tilde{Z}$ does not depend of $X$.

The constant $C_{n}(\alpha)$ has been already computed in [8](Appendix B)

$$
C_{n}(\alpha)=\frac{2 \pi^{(n+1) / 2} \Gamma((\alpha+1) / 2)}{\alpha \sin (\alpha \pi / 2) \Gamma(\alpha) \Gamma((n+\alpha) / 2)} .
$$

We have proved that

$$
I_{n}(X)=C_{n}(\alpha)\|X\|^{\alpha}
$$

For any $\theta, \theta^{\prime}$, one has

$$
\left|1-\mathrm{e}^{\mathrm{i} \theta}\right|^{2}+\left|1-\mathrm{e}^{-\mathrm{i} \theta^{\prime}}\right|^{2}-\left|1-\mathrm{e}^{\mathrm{i}\left(\theta^{\prime}-\theta\right)}\right|^{2}=\left(1-\mathrm{e}^{\mathrm{i} \theta}\right)\left(1-\mathrm{e}^{-\mathrm{i} \theta^{\prime}}\right)+\left(1-\mathrm{e}^{-\mathrm{i} \theta}\right)\left(1-\mathrm{e}^{\mathrm{i} \theta^{\prime}}\right) .
$$

Taking $\alpha=H(X)+H\left(X^{\prime}\right)$, one gets

$$
R_{n}\left(X, X^{\prime}\right)=\frac{C_{n}\left(H(X)+H\left(X^{\prime}\right)\right)}{2}\left(\|X\|^{H(X)+H\left(X^{\prime}\right)}+\left\|X^{\prime}\right\|^{H(X)+H\left(X^{\prime}\right)}-\left\|X-X^{\prime}\right\|^{H(X)+H\left(X^{\prime}\right)}\right) .
$$

\section{2. $\ell^{2}$-space}

Let us define, for $0<\alpha<2$

$$
F(\alpha)=\frac{2 \Gamma((\alpha+1) / 2)}{\alpha \sin ((\alpha \pi) / 2) \Gamma(\alpha)}
$$

Set, for $0<\alpha<2$

$$
v_{n}(\alpha)=\pi^{-n / 4} \mathrm{e}^{-n / 4} 2^{n / 4+\alpha / 2+1 / 4} n^{n / 4+\alpha / 2-1 / 4} .
$$

Let $H$ be a function from $\ell^{2}$ into $(0,1)$. 


\section{Proposition 2.2.}

- The function

$$
R\left(X, X^{\prime}\right)=\frac{F\left(H(X)+H\left(X^{\prime}\right)\right)}{2}\left(\|X\|^{H(X)+H\left(X^{\prime}\right)}+\left\|X^{\prime}\right\|^{H(X)+H\left(X^{\prime}\right)}-\left\|X-X^{\prime}\right\|^{H(X)+H\left(X^{\prime}\right)}\right)
$$

is the covariance function of a centered Gaussian field $B$ indexed by $\ell^{2}$,

- the restriction of this Gaussian field $B$ to $\mathbb{R}^{n}$ is not the Gaussian field $B_{n}$ of Proposition 2.1;

- let $p \leq n$. The field $v_{n}(H(X)) B_{n}(X), X \in \mathbb{R}^{p}$ converges in distribution, as $n \rightarrow+\infty$, in the sense of finite dimensional margins, to the field $B(X), X \in \mathbb{R}^{p}$.

Proof. Let us prove that $R$ is non-negative definite. Using the Stirling formula

$$
\Gamma(z)=\sqrt{\frac{2 \pi}{z}}\left(\frac{z}{e}\right)^{z}(1+o(1)) \text { as } z \rightarrow+\infty,
$$

little algebra leads to

$$
\lim _{n \rightarrow+\infty} v_{n}(H(X)) v_{n}\left(H\left(X^{\prime}\right)\right) R_{n}\left(X, X^{\prime}\right)=R\left(X, X^{\prime}\right) .
$$

Let $p \leq n$. Using Proposition 2.1, $R_{n}\left(X, X^{\prime}\right), X, X^{\prime} \in \mathbb{R}^{p}$ is the covariance function of a Gaussian field $B(X), X \in \mathbb{R}^{p}$. Therefore, $v_{n}(H(X)) v_{n}\left(H\left(X^{\prime}\right)\right) R_{n}\left(X, X^{\prime}\right)$ is the covariance function of the field $v_{n}(H(X)) B(X)$. By $(2.2), R\left(X, X^{\prime}\right), X, X^{\prime} \in \mathbb{R}^{p}$ is non-negative definite for any $p$. Since $\left(X, X^{\prime}\right) \mapsto R\left(X, X^{\prime}\right), X, X^{\prime} \in \ell^{2}$ is a continuous function, $\left(X, X^{\prime}\right) \mapsto R\left(X, X^{\prime}\right), X, X^{\prime} \in \ell^{2}$ is therefore non-negative definite.

Since the ratio $C_{n}(\alpha) / F(\alpha)$ clearly depends on $n$, the restriction to $\mathbb{R}^{n}$ of the Gaussian field of Proposition 2.2 is not the Gaussian field of Proposition 2.1.

\section{Multifractional Brownian fields indexed by metric spaces With Distances OF NEGATIVE TYPE}

Let $(E, d)$ be a metric space. Let us assume from now on that distance $d$ is of negative type (e.g. [1]), i.e. $\forall n \geq 2, \forall M_{1}, \ldots, M_{n} \in E, \forall \lambda_{1}, \ldots, \lambda_{n} \in \mathbb{R}$ such that $\sum_{1}^{n} \lambda_{i}=0$

$$
\sum_{i, j=1}^{n} \lambda_{i} \lambda_{j} d\left(M_{i}, M_{j}\right) \leq 0 .
$$

Let us recall that $d$ being of negative type is equivalent to the existence of Lévy-Brownian field indexed by $E$ [9]. The Euclidean norms [4], geodesic distances on the spheres [9], the hyperbolic spaces [5] and the real trees [15] are examples of distances of negative type. Another consequence of $d$ being of negative type (see [1]) is the existence of a Hilbert space $\mathcal{H}$ and a continuous mapping $\tilde{F}$ from $E$ into $\mathcal{H}$ such that

$$
d(M, N)=\|\tilde{F}(M)-\tilde{F}(N)\|_{\mathcal{H}}^{2} \quad \forall M, N \in E,
$$

and $\operatorname{span}\left\{\tilde{F}(M)-\tilde{F}\left(M_{0}\right), M \in E\right\}$ is dense in $\mathcal{H}$ for all $M_{0} \in E$. Assume from now on that $\mathcal{H}$ is separable.

Fractional Brownian field indexed by the metric space $E$ has been defined [10] as the centered Gaussian field with covariance function

$$
R(M, N)=\frac{1}{2}\left(d^{2 H}(O, M)+d^{2 H}(O, N)-d^{2 H}(M, N)\right),
$$


where $O$ is a given point of $E$. Since the distance $d$ is of negative type, fractional Brownian field exists at least for $0<H \leq 1 / 2$. It may not exist for $H>1 / 2$ : so is the case for spheres and hyperbolic spaces. The aim of the next theorem is to construct a multifractional Brownian field indexed by $E$. Function $F$ is still defined, for $0<\alpha<2$, by

$$
F(\alpha)=\frac{2 \Gamma((\alpha+1) / 2)}{\alpha \sin ((\alpha \pi) / 2) \Gamma(\alpha)} .
$$

Let $H$ be a function from $E$ into $(0,1 / 2)$, and $O$ be a given point of $E$.

\section{Theorem 3.1.}

- The function

$$
R(M, N)=\frac{F(2(H(M)+H(N)))}{2}\left(d^{H(M)+H(N)}(O, M)+d^{H(M)+H(N)}(O, N)-d^{H(M)+H(N)}(M, N)\right)
$$

is the covariance function of a centered Gaussian field $X(M), M \in E$;

- assume moreover that function $H$ is Lipschitz at point $M$, i.e. there exists $k>0$ such that, for all $N$ in a neighborhood of $M$

$$
|H(M)-H(N)| \leq k d(M, N) .
$$

Let $M$ be an accumulation point of $E$ and let $M_{n} \rightarrow M$. Then $\frac{X\left(M_{n}\right)-X(M)}{d^{H(M)}\left(M_{n}, M\right)}$ converges in distribution, as $n \rightarrow+\infty$, to a centered Gaussian variable with variance $F(4 H(M))$.

Remark 3.2. Since $(E, d)$ is a metric space, and not necessarily a manifold, one can not expect a local asymptotical self-similarity property, as defined in [12].

Proof. Let $\left(e_{i}\right)_{i \geq 1}$ be an orthonormal basis of $\mathcal{H}$. For any $M \in E, \tilde{F}(M)$ can be expanded on the $\left(e_{i}\right)_{i \geq 1}$

$$
\tilde{F}(M)=\sum_{i \geq 1} f_{i}(M) e_{i}
$$

where $f(M)=\left(f_{1}(M), \ldots, f_{n}(M), \ldots\right) \in \ell^{2}$. It follows

$$
\begin{aligned}
d(M, N) & =\|\tilde{F}(M)-\tilde{F}(N)\|_{\mathcal{H}}^{2} \\
& =\|f(M)-f(N)\|^{2} .
\end{aligned}
$$

This leads to

$$
\begin{aligned}
R(M, N)= & \frac{F(2(H(M)+H(N)))}{2}\left(\|f(M)-f(O)\|^{2(H(M)+H(N))}\right. \\
& \left.+\|f(M)-f(O)\|^{2(H(M)+H(N))}-\|f(M)-f(N)\|^{2(H(M)+H(N))}\right) .
\end{aligned}
$$

By (2.1), the covariance $R$ is non-negative definite for any function $H$. Letting $\tilde{H}(f(M))=H(M)$, we can apply Proposition 2.1 to (3.3) and (3.3) defines a non-negative definite function.

Let us now prove the second item of the theorem.

$$
\begin{aligned}
\mathbb{E}\left(X\left(M_{n}\right)-X(M)\right)^{2}= & R\left(M_{n}, M_{n}\right)+R(M, M)-2 R\left(M, M_{n}\right) \\
& T_{1}+T_{2}+T_{3} \\
T_{1}= & \left(F\left(4 H\left(M_{n}\right)\right)-F\left(2 H\left(M_{n}\right)+2 H(M)\right)\right) \\
& \times d^{H\left(M_{n}\right)+H(M)}\left(O, M_{n}\right), \\
T_{2}= & \left(F\left(4 H\left(M_{n}\right)\right)-F\left(2 H\left(M_{n}\right)+2 H(M)\right)\right) \\
& \times d^{H\left(M_{n}\right)+H(M)}(O, M), \\
T_{3}= & F\left(2 H\left(M_{n}\right)+2 H(M)\right) d^{H\left(M_{n}\right)+H(M)}\left(M, M_{n}\right) .
\end{aligned}
$$


Since $H$ is a continuous function, the sequence $d^{H\left(M_{n}\right)+H(M)}\left(O, M_{n}\right)$ is bounded. Function $\alpha \rightarrow F(\alpha)$ is $C^{1}$, and $H$ is a Lipschitz function. There exists $C_{1}$ such that

$$
\left|T_{1}\right| \leq C_{1} d\left(M, M_{n}\right) .
$$

Similar arguments show that there exists $C_{2}$ such that

$$
\left|T_{2}\right| \leq C_{2} d\left(M, M_{n}\right) .
$$

Then

$$
d^{H\left(M_{n}\right)-H(M)}\left(M_{n}, M\right)=\exp \left\{\frac{H\left(M_{n}\right)-H(M)}{d\left(M_{n}, M\right)} d\left(M_{n}, M\right) \log d\left(M_{n}, M\right)\right\} .
$$

One gets

$$
\lim _{n \rightarrow+\infty} d^{H\left(M_{n}\right)-H(M)}\left(M_{n}, M\right)=1,
$$

and

$$
\begin{aligned}
\lim _{n \rightarrow+\infty} \mathbb{E}\left(\frac{X\left(M_{n}\right)-X(M)}{d^{H(M)}\left(M, M_{n}\right)}\right)^{2} & =\lim _{n \rightarrow+\infty} \frac{T_{3}}{d^{2 H(M)}\left(M, M_{n}\right)} \\
& =F(4 H(M)) .
\end{aligned}
$$

Acknowledgements. The author would like to thank the anonymous referees for valuable comments and suggestions.

\section{REFERENCES}

[1] B. Bekka, P. De la Harpe and A. Valette, Kazhdan's property (T). Cambridge University Press (2008).

[2] A. Benassi, S. Jaffard and D. Roux, Gaussian processes and pseudodifferential elliptic operators. Rev. Mat. Iberoam. 13 (1997) 19-90.

[3] A. Benassi, S. Cohen and J. Istas, Identifying the multifractional function of a Gaussian process. Stat. Probab. Lett. 39 (1998) 337-345.

[4] N. Chentsov, Lévy's Brownian motion of several parameters and generalized white noise. Theory Probab. Appl. 2 (1957) 265-266.

[5] J. Faraut and H. Harzallah, Distances hilbertiennes invariantes sur un espace homogène. Ann. Inst. Fourier 24 (1974) $171-217$.

[6] A. Kolmogorov, Wienersche Spiralen und einige andere interessante Kurven im Hilbertsche Raum (German). C. R. (Dokl.) Acad. Sci. URSS 26 (1940) 115-118.

[7] C. Lacaux, Real harmonizable multifractional Lévy motions. Ann. Inst. Henri Poincaré 40 (2004) $259-277$.

[8] C. Lacaux, Series representation and simulation of multifractional Lévy motions. Adv. Appl. Probab. 36 (2004) $171-197$.

[9] P. Lévy, Processus stochastiques et mouvement Brownien. Gauthier-Villars (1965).

[10] J. Istas, Spherical and hyperbolic fractional Brownian motion. Electron. Commun. Probab. 10 (2005) $254-262$.

[11] J. Istas, On fractional stable fields indexed by metric spaces. Electron. Commun. Probab. 11 (2006) $242-251$.

[12] J. Istas and C. Lacaux, On locally self-similar fractional random fields indexed by a manifold. Preprint (2009).

[13] B. Mandelbrot and J. Van Ness, Fractional Brownian motions, fractional noises and applications. SIAM Review 10 (1968) 422-437.

[14] R. Peltier and J. Lévy-Vehel, Multifractional Brownian motion: definition and preliminary results. Rapport de recherche de l'INRIA 2645 (1996).

[15] A. Valette, Les représentations uniformément bornées associées à un arbre réel. Bull. Soc. Math. Belgique 42 (1990) $747-760$. 